\title{
MARKOVIAN SOLUTIONS OF INVISCID BURGERS EQUATION
}

\author{
MARIE-LINE CHABANOL AND JEAN DUCHON
}

\begin{abstract}
For solutions of (inviscid, forceless, one dimensional) Burgers equation with random initial condition, it is heuristically shown that a stationary Feller-Markov property (with respect to the space variable) at some time is conserved at later times, and an evolution equation is derived for the infinitesimal generator. Previously known explicit solutions such as FrachebourgMartin's (white noise initial velocity) and Carraro-Duchon's Lévy process intrinsic-statistical solutions (including Brownian initial velocity) are recovered as special cases.
\end{abstract}

\section{INTRODUCTION}

We consider the inviscid Burgers equation $\partial_{t} u+\partial_{x}\left(\frac{1}{2} u^{2}\right)=0$ with random initial data $u_{0}$. Burgers equation has originally been introduced 2] as a $1 \mathrm{D}$ model of turbulence. Although it is now clear that it does not exhibit lots of features of "true" turbulence, we nevertheless still think it is a good equation on which one can try and find new methods to apply on Euler equation. Having this in mind, taking random initial data seems quite a natural problem. It is also physically relevant in the contexts of interface dynamics, of aggregation of particles [ $[$, and some others. Burgers equation with a random force on the r.h.s. has also been studied, mainly as a "benchmark" to test methods designed for (Navier-Stokes) forced turbulence, many of which turn out to produce spurious predictions when applied to the simpler Burgers case. See 5 for instance.

The case of a Brownian initial data has already been investigated by Sinai [11. Carraro and Duchon [3, 4] showed that Lévy processes are conserved by Burgers equation. They also obtained the explicit evolution equation for the characteristic function of the Lévy process solutions of Burgers. A noticeable point is that they made no use of the Hopf-Cole construction of the solution (Bertoin [1] recovered essentially the same result with Hopf-Cole).

We will essentially follow Carraro and Duchon : first we define what we call a statistical solution of Burgers equation, and write an infinite set of equations for the $n$-point functions of such solutions. We show that the assumption that the process is Feller (in space) for all time yields an evolution equation for the infinitesimal generator of this process. Conversely, a Feller process whose generator satisfies this equation is a statistical solution of Burgers equation. This will allow us to recover

Date: October 11, 2018.

1991 Mathematics Subject Classification. Primary: 35Q53; Secondary: 60J25.

Key words and phrases. Burgers, inviscid, turbulence, Markov.

The authors thank Christophe Giraud for fruitful discussions, the Erwin Schrödinger Institute in Vienna for their invitation and support, the organizers of the program "developed turbulence" there (Gawȩdzki, Kupiainen, Vergassola) and also Uriel Frisch and Yakov Sinai who conducted the workshop "Burgers turbulence and beyond". 
Carraro and Duchon's result on Lévy processes, as a special case. Frachebourg and Martin's explicit solution [7] in the case of an initial white noise velocity is also a particular solution to our equation.

\section{Notations AND DEFINitions}

A Markov process $u(x)_{x \in \mathbf{R}}$ can be characterized by its one point and its transition probabilities $p_{x}(d u)$ and $q_{x, y}(u, d v), x<y$, that satisfy, $\forall x_{0}<\cdots<x_{k}$ and $f_{i}$ borelian positive, $\quad \mathbf{E}\left[\prod_{i=0}^{k} f_{i}\left(u\left(x_{i}\right)\right)\right]=$

$$
\int p_{x_{0}}\left(d u_{0}\right) f_{0}\left(u_{0}\right) \int q_{x_{0}, x_{1}}\left(u_{0}, d u_{1}\right) f_{1}\left(u_{1}\right) \cdots \int q_{x_{k-1}, x_{k}}\left(u_{k-1}, d u_{k}\right) f_{k}\left(u_{k}\right) .
$$

A Markov process is homogeneous if its transition probabilities $q_{x, y}$ depend on $x$ and $y$ only through $y-x$. In this case, we write $q_{h}$ instead of $q_{x, x+h}$.

A process $u(x)_{x \in \mathbf{R}}$ is stationary if and only if it is translation invariant : the law of $\left(u_{x+x_{1}}, \ldots, u_{x+x_{n}}\right)$ does not depend on $x$. Hence a Markov process is stationary if and only if it is homogeneous and its one point probability $p_{x}(d u)$ does not depend on $x$.

If $u$ is a homogeneous Markov process, $h>0$, and $f$ is a continuous function vanishing at infinity, we put $Q_{h} f(u)=\int f(v) q_{h}(u, d v)$.

A Feller process is a homogeneous Markov process such that for each $f$, for each $h>0, Q_{h} f$ is also continuous and vanishes at infinity, and $\lim _{h \rightarrow 0} Q_{h} f=f$ pointwise.

A Feller process always has a càdlàg version 9 .

One can define the infinitesimal generator of a Feller process : it is the operator $A$, defined for all the functions $f$ such that the limit below exists, by

$$
\forall x \in \mathbf{R}, A f(x)=\lim _{h \rightarrow 0^{+}} \frac{Q_{h} f(x)-f(x)}{h}
$$

Formally, $Q_{h}=\exp (h A), Q_{h}^{\prime}:=d Q / d h=A Q_{h}$ and an invariant measure $p_{0}$ satisfies ${ }^{t} A p_{0}=0$.

The

\section{Statistical solutions of Burgers equation}

We will closely follow [4] (see also [10). Let $E$ be the space of càdlàg real functions equipped with the smallest $\sigma$-algebra $\mathcal{C}(E)$ such that for each $x \in \mathbf{R}$, $u \mapsto u(x)$ is measurable. Let $\mathcal{D}$ be the set of real $C^{\infty}$ functions with compact support. A probability $\mu$ on $E$ is then characterized by its characteristic function

$$
v \in \mathcal{D} \mapsto \int_{E} \exp \left[i \int_{\mathbf{R}} u(x) v(x) d x\right] d \mu(u)=\hat{\mu}(v) .
$$

Let $u_{0}:(\Omega, \mathcal{A}, P) \rightarrow E$ be a random process, defined on some probability space, and let $\mu_{0}: \mathcal{C}(E) \rightarrow[0,1]$ denote its probability law. Assume $u(x, t)$ is a (weak) solution of Burgers equation with $u(., 0)=u_{0}, u(., t) \in E$ for $t>0$, and everything makes sense in the following calculation : integrability, and differentiability with respect to $t$. Let $\mu_{t}$ denote the law of $u(., t)$. Formally, one then gets for each 
$v \in \mathcal{D}:$

$$
\begin{aligned}
\partial_{t} \hat{\mu}_{t}(v) & =\int_{E} \partial_{t}\left\{\exp \left[i \int_{\mathbf{R}} u(x) v(x) d x\right]\right\} d \mu_{t}(u) \\
& =\int_{E} \partial_{t}\left\{\exp \left[i \int_{\mathbf{R}} u(x, t) v(x) d x\right]\right\} d \mu_{0}\left(u_{0}\right) \\
& \left.=\int_{E} \exp \left[i \int_{\mathbf{R}} u(x, t) v(x) d x\right)\right] \partial_{t}\left[i \int_{\mathbf{R}} u(x, t) v(x) d x\right] d \mu_{0}\left(u_{0}\right) \\
& =\int_{E} \exp \left[i \int_{\mathbf{R}} u(x, t) v(x) d x\right] i \int_{\mathbf{R}} \frac{1}{2} u(x, t)^{2} v^{\prime}(x) d x d \mu_{0}\left(u_{0}\right) \\
& =i \int_{E} \int_{\mathbf{R}} \frac{1}{2} u(x)^{2} v^{\prime}(x) d x \exp \left[i \int_{\mathbf{R}} u v\right] d \mu_{t}(u)
\end{aligned}
$$

Hence our definition of a statistical solution of Burgers equation :

Definition 1. A statistical solution of Burgers equation is a set $\left(\mu_{t}\right)_{t \geq 0}$ of probabilities on $(E, \mathcal{C}(E))$ such that for any $v \in \mathcal{D}$,

$$
\partial_{t} \hat{\mu}_{t}(v)=i \int_{E} \int_{\mathbf{R}} \frac{1}{2} u(x)^{2} v^{\prime}(x) d x \exp \left[i \int_{\mathbf{R}} u v\right] d \mu_{t}(u)
$$

Let us assume now that we have a statistical solution of Burgers equation, $\left(\mu_{t}\right)_{t \geq 0}$, and that for all $t$, all the moments of $\mu_{t}$ are well defined. Then one can write

$$
\exp \left[i \int_{\mathbf{R}} u(x) v(x) d x\right]=\sum_{n=0}^{\infty} \frac{i^{n}}{n !} \int_{\mathbf{R}^{n}} \prod_{j=1}^{n} u\left(x_{j}\right) v\left(x_{j}\right) d x_{j}
$$

Equation (11) thus becomes $\forall v \in \mathcal{D}$,

$$
\begin{aligned}
2 \sum_{n=0}^{\infty} \frac{i^{n}}{n !} \int_{\mathbf{R}^{n}} \partial_{t} \mathbf{E}\left[\prod_{j=1}^{n} u\left(x_{j}\right) v\left(x_{j}\right)\right] \prod d x_{j}= \\
=i \sum_{n=0}^{\infty} \frac{i^{n}}{n !} \int_{\mathbf{R}^{n+1}} \mathbf{E}\left[\prod_{j=1}^{n} u\left(x_{j}\right) v\left(x_{j}\right) u(x)^{2} v^{\prime}(x)\right] d x \prod d x_{j}= \\
\quad=i \sum_{n=0}^{\infty} i^{n} \int_{x_{0}<x_{1}<\ldots<x_{n}} \sum_{j=0}^{n} \mathbf{E}\left[u\left(x_{j}\right) \frac{v^{\prime}\left(x_{j}\right)}{v\left(x_{j}\right)} \prod_{k=0}^{n} u\left(x_{k}\right) v\left(x_{k}\right)\right] \prod d x_{k}
\end{aligned}
$$

\section{Evolution EQUation for Markov solutions}

We are now looking for solutions such that at each time $t, x \mapsto u(x, t)$ is a stationary Feller process (with respect to space $x$ ). We are going to show that for such processes, the infinite set of equations (2) is equivalent to an evolution equation for the infinitesimal generator of $u$.

We thus assume now that the solution $x \mapsto u(x, t)$ is a stationary Feller process, with one point probability $p(d u, t)$ and transition probability $q_{h}\left(u_{1}, d u_{2}, t\right)$, the equation (2) becomes $\forall v \in \mathcal{D}$ : 


$$
\begin{aligned}
2 \sum_{n=1}^{\infty} \int_{x_{1}<\cdots<x_{n}} d x_{1} \ldots d x_{n} u_{1} v\left(x_{1}\right) \ldots u_{n} v\left(x_{n}\right) \\
\quad \times \partial_{t}\left[p\left(d u_{1}\right) q_{h_{2}}\left(u_{1}, d u_{2}\right) \ldots q_{h_{n}}\left(u_{n-1}, d u_{n}\right)\right] \\
=i \sum_{n=1}^{\infty} i^{n} \int_{x_{0}<\cdots<x_{n}} d x_{0} \ldots d x_{n} p\left(d u_{0}\right) q_{h_{1}}\left(u_{0}, d u_{1}\right) \ldots q_{h_{n}}\left(u_{n-1}, d u_{n}\right) \\
\quad \times\left[u_{0} \frac{v^{\prime}}{v}\left(x_{0}\right)+\cdots+u_{n} \frac{v^{\prime}}{v}\left(x_{n}\right)\right] u_{0} v\left(x_{0}\right) \ldots u_{n} v\left(x_{n}\right) \\
=i \sum_{n=1}^{\infty} i^{n} \int_{x_{0}<\cdots<x_{n}} d x_{0} \ldots d x_{n} u_{0} v\left(x_{0}\right) \ldots u_{n} v\left(x_{n}\right) \\
\quad \times p\left(d u_{0}\right) q_{h_{1}}\left(u_{0}, d u_{1}\right) \ldots q_{h_{n}}\left(u_{n-1}, d u_{n}\right) \\
\quad \times\left\{u_{1} \frac{q_{h_{2}}^{\prime}}{q_{h_{2}}}\left(u_{1}, d u_{2}\right)+u_{2}\left[\frac{q_{h_{3}}^{\prime}}{q_{h_{3}}}\left(u_{2}, d u_{3}\right)-\frac{q_{h_{2}}^{\prime}}{q_{h_{2}}}\left(u_{1}, d u_{2}\right)\right]+\ldots\right. \\
\left.+u_{n-1}\left[\frac{q_{h_{n}}^{\prime}}{q_{h_{n}}}\left(u_{n-1}, d u_{n}\right)-\frac{q_{h_{n-1}}^{\prime}}{q_{h_{n-1}}}\left(u_{n-2}, d u_{n-1}\right)\right]-u_{n} \frac{q_{h_{n}}^{\prime}}{q_{h_{n}}}\left(u_{n-1}, d u_{n}\right)\right\}
\end{aligned}
$$

(by integrating by parts; we note $h_{j}=x_{j}-x_{j-1}$ and $q_{h}^{\prime}=\partial q_{h} / \partial h$ ).

This equality is equivalent to the following infinite set of equations : $\forall n \in$ $\mathbf{N}^{*}, \forall x_{1}<\ldots<x_{n}$ :

$$
\begin{aligned}
& 2 \partial_{t} \mathbf{E}\left[u\left(x_{1}\right) \ldots u\left(x_{n}\right)\right]= \\
& \quad \int u_{1} \ldots u_{n} p\left(d u_{1}\right) q_{h_{2}}\left(u_{1}, d u_{2}\right) \ldots q_{h_{n}}\left(u_{n-1}, d u_{n}\right) \\
& \quad \times\left\{u_{1} \frac{q_{h_{2}}^{\prime}}{q_{h_{2}}}\left(u_{1}, d u_{2}\right)+u_{2}\left[\frac{q_{h_{3}}^{\prime}}{q_{h_{3}}}\left(u_{2}, d u_{3}\right)-\frac{q_{h_{2}}^{\prime}}{q_{h_{2}}}\left(u_{1}, d u_{2}\right)\right]+\ldots\right. \\
& \left.+u_{n-1}\left[\frac{q_{h_{n}}^{\prime}}{q_{h_{n}}}\left(u_{n-1}, d u_{n}\right)-\frac{q_{h_{n-1}}^{\prime}}{q_{h_{n-1}}}\left(u_{n-2}, d u_{n-1}\right)\right]-u_{n} \frac{q_{h_{n}}^{\prime}}{q_{h_{n}}}\left(u_{n-1}, d u_{n}\right)\right\}
\end{aligned}
$$

One then gets the evolution equations for $p, q$ and $A$ by taking limits in which some of the $x_{i}$ s are equal. If one makes every $x_{i}$ tend to $x_{1}$, the preceding set of equations gives formally, $\forall n \in \mathbf{N}^{*}$ :

$$
2 \int \partial_{t} p(d u) u^{n}=\int p(d u)\left(-U A U^{n}+U^{n} A U\right)(u)
$$

where $U^{n}$ denotes the function $u \mapsto u^{n}$.

If one makes some of the $x_{i}$ s tend to $x_{1}$, and the others tend to $x_{2}=x_{1}+h$, one then gets $\forall n \in \mathbf{N}^{*}, \forall k<n, \forall x_{1} \in \mathbf{R}, \forall h \in \mathbf{R}^{+*}$ :

$$
\begin{aligned}
& 2 \partial_{t} \mathbf{E}\left[u\left(x_{1}\right)^{k} u\left(x_{1}+h\right)^{n-k}\right]=\int p(d u)\left\{-U A\left(U^{k} Q_{h} U^{n-k}\right)+U^{k+1} Q_{h} A U^{n-k}\right. \\
& \left.+U^{k}\left[A\left(U Q_{h} U^{n-k}\right)-A Q_{h} U^{n-k+1}+Q_{h}\left(U^{n-k} A U\right)-Q_{h}\left(U A U^{n-k}\right)\right]\right\}(u)
\end{aligned}
$$


One then easily finds, if $\eta$ is in the domain of $A$ :

$$
\begin{aligned}
2 \int \partial_{t} p(d u) \eta(u)= & \int p(d u)[-u A \eta(u)+\eta(u) A U(u)] \\
2 \partial_{t} Q_{h} \eta= & U A Q_{h} \eta+A\left(U Q_{h} \eta\right)-Q_{h}(U A \eta) \\
& -A Q_{h}(U \eta)+Q_{h}(\eta A U)-A U Q_{h} \eta
\end{aligned}
$$

These two equalities sum up into one : $\forall \eta$ in the domain of $A$,

$$
2 \partial_{t} A \eta=U A^{2} \eta-A^{2}(U \eta)+A(\eta A U)-A U A \eta
$$

or, introducing the operators $M_{U}$ and $M_{A U}$ defined as $M_{U} \eta(u)=u \eta(u)$ and $M_{A U} \eta(u)=A U(u) \eta(u)$ :

$$
2 \partial_{t} A=M_{U} A^{2}-A^{2} M_{U}+A M_{A U}-M_{A U} A
$$

If this latter equality holds, one can easily check that if ${ }^{t} A p=0$ for all time, then $p$ verifies (6), and $Q_{h}=\exp (h A)$ verifies (17).

Hence a Feller statistical solution of (2) is solution of (6) and (7), which are equivalent to (91).

Conversely, it is a matter of simple algebra to check that (6) and (7) imply (2) : indeed one can then write for any $x_{1}<\cdots<x_{n}\left(\right.$ recall $\left.h_{i}=x_{i}-x_{i-1}\right)$ :

$$
\begin{gathered}
2 \partial_{t} E\left[u\left(x_{1}\right) \ldots u\left(x_{n}\right)\right]=2 \partial_{t} \int p(d u) M_{U} Q_{h_{2}} \ldots M_{U} Q_{h_{n}} U(u) \\
=2 \int \partial_{t} p(d u) M_{U} Q_{h_{2}} \ldots M_{U} Q_{h_{n}} U(u) \\
+2 \sum_{j=2}^{n} \int p(d u) M_{U} Q_{h_{2}} \ldots M_{U} Q_{h_{j-1}} M_{U} \partial_{t} Q_{h_{j}} M_{U} Q_{h_{j+1}} \ldots M_{U} Q_{h_{n}} U(u) \\
=\int p(d u) u\left[-A M_{U} Q_{h_{2}} M_{U} \ldots Q_{h_{n}} U(u)+Q_{h_{2}} M_{U} \ldots Q_{h_{n}} U(u) A U(u)\right] \\
+\sum_{j=2}^{n} \int p(d u) M_{U} Q_{h_{2}} \ldots M_{U}\left[M_{U} A Q_{h_{j}} \eta_{j}+A\left(U Q_{h_{j}} \eta_{j}\right)-Q_{h_{j}}\left(U A \eta_{j}\right)\right. \\
\left.-A Q_{h_{j}}\left(U \eta_{j}\right)+Q_{h_{j}}\left(\eta_{j} A U\right)-A U A \eta_{j}\right]
\end{gathered}
$$

where $\eta_{j}=M_{U} Q_{h_{j+1}} \ldots M_{U} Q_{h_{n}} U$. Many terms cancel, one gets

$$
=\int p(d u) u \sum_{j=2}^{n} Q_{h_{2}} M_{U} \ldots Q_{h_{j-1}} M_{U}\left[M_{U} Q_{h_{j}}^{\prime}-Q_{h_{j}}^{\prime} M_{U}\right] \eta_{j}
$$

which is just one integration by parts away from (2).

Therefore, if $u(x, t)$ is a Feller process, it is a statistical solution of Burgers if and only if its infinitesimal generator is solution of (9). In some sense, the Feller assumption yields an exact closure of the infinite set (2). Of course, nothing guarantees the existence of solutions of (9), although we show later that the Brownian and white noise initial cases give formal solutions to it. Nevertheless, a close look at Bertoin's proof using Hopf-Cole [1] makes us strongly suspect that the absence of positive jumps may be essential to guarantee the existence of solutions. This would also be reasonable from a physical point of view : solutions with positive jumps are unphysical. 


\section{The CASE OF LÉVY PROCESSES}

We will see how one can recover formally the results of 4. The initial velocity $u_{0}$ is here supposed to be a Lévy process (which means that it has independent and stationary increments) of finite variance having no negative jumps. This covers in particular the case of $u_{0}$ Brownian. Such processes are characterized by their second exponent $\phi$, defined by $\forall x<y, \forall \lambda \in \mathbf{R}^{+}$:

$$
E\left\{\exp \left[\lambda\left(u_{0}(y)-u_{0}(x)\right)\right]\right\}=\exp [(y-x) \phi(\lambda)]
$$

A Lévy process can be considered as a limit case of stationary Markov process (the one point distribution $p$ is replaced with Lebesgue measure). One can also formally define an infinitesimal generator by the relations : $\forall \lambda \in \mathbf{R}^{+}$,

$$
A e_{\lambda}=\phi(\lambda) e_{\lambda}
$$

where we have noted $e_{\lambda}$ the function $u \mapsto \exp (\lambda u)$ (which of course is not in the domain of $A \ldots)$. One can inject these relations into the evolution equation (9). Using $u e_{\lambda}(u)=\partial_{\lambda} e_{\lambda}(u)$, and $A U=$ constant, one gets an evolution equation for $\phi$; it turns out that this equation is also the Burgers equation :

$$
2 \partial_{t} \phi(\lambda)=-\partial_{\lambda} \phi^{2}
$$

Carraro and Duchon [4] have checked that if $\phi_{0}$ is the exponent of a Lévy process of finite variance with negative jumps, (11) has a smooth solution for all time $t \geq 0$, which is still the exponent of a homogeneous Lévy process with negative jumps.

Hence such Lévy processes are conserved by the Burgers equation. The Brownian case corresponds to $\phi_{0}(\lambda)=\lambda^{2} / 2$, and this yields $\phi(\lambda, t)=(1+\lambda t-\sqrt{1+2 \lambda t}) / t^{2}$.

\section{Evolution EquATion FOR THE JUMP PROCESS}

The infinitesimal generator of an arbitrary Markov process can be written as the sum of three terms (see [9]) : a diffusion term, a drift term and a jump term :

$$
A f(x)=a(x) f^{\prime \prime}(x)+b(x) f^{\prime}(x)+\int n(x, d y)(f(y)-f(x))
$$

The measure $n(x, d y)$ represents the jump part of the process : it gives the number of jumps going from $x$ to $y$. In our case, all these coefficients will of course depend on time. To write an evolution equation for $n$, we assume $b=1 / t$ and $a=0$ for $t>0$, and all jumps are negative. Equation (9) then yields an evolution equation for $n: \forall x>y$,

$$
\begin{aligned}
2 \partial_{t} n(x, d y, t) & =\frac{1}{t}(x-y)\left(\partial_{x} n(x, d y, t)-\partial_{y} n(x, d y, t)\right) \\
+\int_{-\infty}^{x} n(x, d u, t)[(x-y) n(u, d y, t) & +(y-u) n(x, d y, t)] \\
& -\int_{-\infty}^{y}(x-u) n(y, d u, t) n(x, d y, t)
\end{aligned}
$$

\section{The CASE OF AN INITIAL White NOISE PROCESS}

Frachebourg and Martin [7] have investigated the case of an initial white noise velocity. Using the Hopf-Cole construction, they obtain explicit formulas for the laws of $u(x, t)$ and its jumps. They actually rederived results about Brownian motion with a parabolic drift that had been previously established by Groeneboom [8] out 
of the Burgers context. Using Frachebourg and Martin's results or Groeneboom's paper, the infinitesimal generator in the case of an initial white noise process is found to be, in the case where $<u_{0}(x) u_{0}(y)>=(1 / 8) \delta(x-y)$ :

$$
A f(x)=\frac{1}{t} f^{\prime}(x)+4 \int_{-\infty}^{x}(f(y)-f(x))(x-y) \frac{J\left(y t^{1 / 3}\right)}{J\left(x t^{1 / 3}\right)} I\left(x t^{1 / 3}-y t^{1 / 3}\right) d y
$$

where $I$ and $J$ are given by their Fourier and Laplace transforms in terms of the Airy function Ai :

$$
\begin{aligned}
J(u) & =\frac{1}{2 i \pi} \int_{-i \infty}^{i \infty} d z \frac{\exp (u z)}{2^{1 / 3} \operatorname{Ai}\left(2^{-1 / 3} z\right)} \\
2 I(u) & =\left(2 \pi u^{3}\right)^{-1 / 2}+\frac{1}{2 i \pi} \int_{-i \infty}^{i \infty} \exp (u z)\left(\frac{2^{2 / 3} \mathrm{Ai}^{\prime}\left(2^{-1 / 3} z\right)}{\operatorname{Ai}\left(2^{-1 / 3} z\right)}+(2 z)^{1 / 2}\right)
\end{aligned}
$$

We have checked that the evolution equation (9) is indeed verified : it amounts to expressing convolutions like $u I * J, u I * u I, u I * u J$ in terms of $J^{\prime}$ and $I^{\prime}$. It can be done using relations (13) and (14) and the fact that $\operatorname{Ai}^{\prime \prime}(x)=x \operatorname{Ai}(x)$.

\section{Conclusion}

We have heuristically shown that for Feller stationary processes, Burgers equation is equivalent to an evolution equation for their infinitesimal generators. It gives strong evidence that the Feller property is conserved by Burgers equation, although we suspect that the negativity of jumps in the initial velocity should be required. Our evolution equation provides an equation for the jump process, and this might lead to other exact statistical solutions of Burgers equation. The previous exact solutions concerning an initial Brownian or white noise velocity are both particular solutions of our equation.

\section{REFERENCES}

[1] J. Bertorn. The inviscid Burgers equation with Brownian initial velocity. Commun. Math. Phys., 193:397-406, 1998.

[2] J.M. Burgers. The nonlinear diffusion equation. Dordrecht: D. Reidel, 1974.

[3] L. Carraro and J. Duchon. Solutions statistiques intrinsèques de l'équation de Burgers et processus de Lévy. C. R. Acad. Sci., Paris, Sér. I, 319:855-858, 1994.

[4] L. Carraro and J. Duchon. Equation de Burgers avec conditions initiales à accroissements indépendants et homogènes. Ann. Inst. Henri Poincaré Anal. Non Linéaire, 15:431-458, 1998.

[5] W. E and E. vanden EiJnden. Statistical theory for the stochastic Burgers equation in the inviscid limit. Comm. Pure Appl. Math., LIII:852-901, 2000.

[6] L. Frachebourg, P. A. Martin, and J. Piasecki. Ballistic aggregation : a solvable model of irreversible many particle dynamics. Physica A, 279:69, 2000.

[7] L. Frachebourg and P.A. Martin. Exact statistical properties of the Burgers equation. J. Fluid Mech., 417:323-349, 2000.

[8] P. Groeneboom. Brownian motion with a parabolic drift and Airy functions. Probab. Theory Related Fields, 81:79-109, 1989.

[9] D. Revuz and M. Yor. Continuous martingales and Brownian motion. Springer, 1999.

[10] R. Robert. Statistical hydrodynamics. In S. Friedlander and D. Serre, editors, Handbook of mathematical fluid mechanics, volume 2. Elsevier, 2003.

[11] Ya. G. Sinai. Statistics of shocks in solutions of the inviscid Burgers equation. Commun. Math. Phys., 148:601-621, 1992. 
Institut Fourier (Grenoble), UMR 5582 CNRS-Université Joseph Fourier, 100, Rue des Mathématiques, B.P. 74, 38041 Saint-Martin D'Hères CedeX

E-mail address: Marie-Line.Chabanol@ujf-grenoble.fr, Jean.Duchon@ujf-grenoble.fr 